\section{Band 20, Heft 2, Juni 2010}

Editorial

85 Interaktion körperlicher und psychischer Erkrankungen Hohagen, F. (Lübeck)

\section{Übersichtsarbeiten}

86 Metakognitive und andere kognitivverhaltenstherapeutische Verfahren bei posttraumatischer Belastungsstörung Simons, M. (Aachen)

93 Ein langer Weg der Professionalisierung: Die Geschichte des deutschen Psychotherapeutengesetzes Vangermain, D. (Potsdam); Brauchle, G. (Hall/Tirol)

\section{Originalarbeit}

101 Welche Funktionen erfüllen Zwangssymptome? - Ergebnisse einer Analyse im stationären Setting Külz, A.K.; Lumpp, A.; Herbst, N.; Stelzer, N. (Freiburg i.Br.); Förstner, U. (Bad Aussee); Voderholzer, U. (Freiburg i.Br., Prien am Chiemsee)

Originalarbeit - Diagnostik

109 Therapieplanung mithilfe des Interviews zur operationalisierten Fertigkeitsdiagnostik (OFD):

Evaluation an einer klinischen Stichprobe Stenzel, N. (Marburg); Krumm, S. (Köln); Rief, W. (Marburg)

Für die Praxis

119 Integration von Verhaltenstherapie und Neuropsychologie. Ein ambulanter Behandlungsansatz für kognitive und emotional-motivationale Störungen nach erworbenen Hirnschädigungen Exner, C.; Doering, B.K.; Conrad, N.; Rief, W. (Marburg)

127 Integration einer existenziellen Perspektive in die kognitiv-behaviorale Therapie chronischer Schmerzen Gebler, F.A. (Hopfen am See)

\section{Pro und Contra}

136 Ist Vergebung eine Voraussetzung therapeutischer Veränderungen?

Ehlert, U. (Zürich); Knaevelsrud, C. (Berlin); Kämmerer, A. (Heidelberg)

\section{Vol. 20, Issue 2, June 2010}

Editorial

85 Interaction between Physical and Mental Diseases Hohagen, F. (Lübeck)

Review Articles

86 Metacognitive Therapy and Other CognitiveBehavioral Treatments for Posttraumatic Stress Disorder

Simons, M. (Aachen)

93 A Long Way to Professionalism: The History of the German Psychotherapy Law

Vangermain, D. (Potsdam); Brauchle, G. (Hall/Tirol)

\section{Original Article}

101 Functions of Obsessive Compulsive Symptoms - Results of an Inpatient Study Külz, A.K.; Lumpp, A.; Herbst, N.; Stelzer, N. (Freiburg i.Br.); Förstner, U. (Bad Aussee); Voderholzer, U. (Freiburg i.Br., Prien am Chiemsee)

Original Article - Diagnostics

109 Treatment Planning in Psychotherapy by Use of the Operationalized Skills Assessment Inventory: Evaluation in a Clinical Sample Stenzel, N. (Marburg); Krumm, S. (Köln); Rief, W. (Marburg)

For the Practitioner

119 Integrating Neuropsychological and CognitiveBehavioral Treatment Approaches. A Treatment Program for Outpatients with Cognitive and EmotionalMotivational Difficulties after Acquired Brain Injury Exner, C.; Doering, B.K.; Conrad, N.; Rief, W. (Marburg)

127 Integration of an Existential Perspective into Cognitive Behavioral Therapy of Chronic Pain Gebler, F.A. (Hopfen am See)

Pro and Con

136 Is Foregiveness Necessary for Therapeutic Change? Ehlert, U. (Zürich); Knaevelsrud, C. (Berlin); Kämmerer, A. (Heidelberg)

\section{KARGER}

Fax +497614520714

Information@Karger.de

www.karger.com
(C) 2010 S. Karger GmbH, Freiburg 


\section{Band 20, Heft 2, Juni 2010}

Brief an die Herausgeber

140 Körperliche Bestrafungen in der Verhaltenstherapie? Zu Heinrichs N: Ein Klaps kann eine angemessene Disziplinierungsmaßnahme sein. Ein Interview mit Robert Larzelere. Verhaltenstherapie 2010;20:61-64 Watzl, H.; Cohen, R. (Konstanz)

143 Buchbesprechungen

148 Fort- und Weiterbildung

153 Mitteilungen der Verbände

158 Tagungen und Kongresse

U2 Erläuterungen zum Titelbild (2. Umschlagseite) Leibl, C.; Schnabl, J.; Ritter, S. (Prien am Chiemsee)

Einen Ausblick auf den Inhalt der kommenden Hefte finden Sie auf Seite 160.

\section{Vol. 20, Issue 2, June 2010}

Letter to the Editors

140 Physical Punishment in Behavioral Therapy? On Heinrichs N: A Swat May Be an Adequate Disciplinary Consequence. An Interview with Robert Larzelere. Verhaltenstherapie 2010;20:61-64 Watzl, H.; Cohen, R. (Konstanz)

143 Book Reviews

148 Education

153 Information by Behavior Therapy Associations

158 Meetings and Conferences

C2 Explanations on the Title (Inside front cocer) Leibl, C.; Schnabl, J.; Ritter, S. (Prien am Chiemsee)

Forthcoming papers are listed on page 160. 